\title{
Selective Processes that Proceed during Formation of Aluminum Layers near the Phase Equilibrium in a Plasma-Condensate System
}

\author{
V. I. Perekrestov, A. S. Kornyushchenko, and Yu. A. Kosminskaya \\ Sumy State University, ul. Rimskogo-Korsakova 2, Sumy, 40007 Ukraine \\ e-mail:perv@phe.sumdu.edu.ua \\ Received September 25, 2007; in final form, January 29, 2008
}

\begin{abstract}
The mechanisms of pore formation in aluminum condensates produced using a radically new technological approach that is based on self-organized sputtering systems are studied. The main technological parameters of pore formation are found to be determined by the closeness to the phase equilibrium in the plasma-condensate system and by the predominant contribution of structural or field selectivity to atom-byatom assembling of the condensate.
\end{abstract}

PACS numbers: 68.55.-a, 81.15.Cd, 81.07.Bc

DOI: $10.1134 / \mathrm{S} 1063784208100174$

\section{INTRODUCTION}

Pore formation technologies generates constant interest due to the use of high-porosity materials in fuel elements, sensors, catalysts, filters, and other devices. As a rule, the existing methods of porous structure formation are based on multistage technological processes $[1,2]$.

Using a highly volatile substance in the vapor-condensate system, one can produce porous structures rather simply. For example, water vapor condensation conditions that are close to phase equilibrium are known to be easily reached even during the deposition of intense vapor fluxes because of high equilibrium water pressures at temperatures below $273 \mathrm{~K}$. For a sufficiently stationary process, these features favor the rapid formation of porous hoarfrost layers. In this case, the degree of supersaturation near equilibrium can be expressed as the difference between the chemical potentials of the vapor and condensate in the form [3]

$$
\Delta \mu=\left(\Omega_{V}-\Omega_{k}\right) \Delta P+\left(s_{V}-s_{k}\right) \Delta T,
$$

where $\Delta P$ and $\Delta T$ are the deviations of the vapor pressure and temperature from the values corresponding to the point in the vapor-condensate equilibrium curve, respectively; $\Omega_{v}$ and $\Omega_{k}$ are the volumes per particle in the vapor and condensate, respectively; and $s_{V}$ and $s_{k}$ are the entropies of the vapor and condensate, respectively.

Under the same temperature conditions, the equilibrium vapor pressures of the majority of metals are several tens of orders of magnitude lower than the equilibrium water vapor pressure [4]. Making allowance for this feature and using Eq. (1), we can state that it is very difficult to produce porous structures in the metal vapor-condensate system (using small values of $\Delta \mu$ ), since this requires the deposition of the weakest vapor fluxes at the maximum growth surface temperature.

The passage to the plasma-condensate system substantially simplifies the solution of this problem. For example, the contact of the plasma with the growth surface increases the adatom energy, enhancing their reevaporation even at a relatively low substrate temperature.

The purpose of this work is to study the pore formation mechanisms in the aluminum condensates formed at the plasma-condensate interface. The choice of aluminum as the object of inquiry is caused by the fact that the equilibrium pressure of its vapors is only $\sim 10^{-7} \mathrm{~Pa}$ even at the melting temperature [4], which almost excludes pore formation in the vapor-condensate system.

\section{PORE FORMATION CONDITIONS AND EXPERIMENTAL TECHNIQUE}

In the plasma-condensate system, it is wise to replace the atomic bond energies on the growth surface by the effective chemical bond energies $E_{e}=-E_{a}+$ $\left(E_{s} \pm \Delta E\right)$, where $E_{a}$ is the chemical bond energy on the growth surface in the vapor-condensate system, $E_{s}$ is the average energy transferred from the plasma to atoms, and $\Delta E$ is the deviation from $E_{s}$. A decrease in $E_{a}$ to an effective numerical value favors the closest approach to equilibrium, which causes the transition from the accelerated formation of supercritical nuclei due to fluctuations in the diffusion field of adatoms [5] to barrier nucleation [6]. In addition, the atom-by-atom assembling of a condensate near equilibrium is effected via the incorporation of atoms into crystal growth 
monostep folds, lattice defects, of crystal joints, and so on. It is important that the density of such active centers is bounded by a critical bond energy $E_{c}$, below which the probability of the atom-by-atom incorporation into the growth surface is negligibly small [7]. The relatively low density of active centers is a prerequisite for pore formation $[8,9]$. At sufficiently high supersaturations, either statistical deposition of a substance or condensate formation via the accelerated formation of polyatomic critical nuclei can occur $[10,11]$. In these two processes, almost any point in the growth surface can serve as a basis for the attachment of a condensed substance irrespective of the structural state of the surface, which excludes selective processes.

The causes of the spatially distributed selectivity in the condensate growth are not restricted to the structural state of the growth surface. It should be noted that, near equilibrium, the curvature of the growth surface both directly affects $\Delta \mu$ [3] and specifies (in the presence of a bias voltage across a substrate holder) electric field fluctuations over the growth surface, which also redistributes the deposited ion fluxes. Hereafter, the possible self-amplifying local condensate growth in regions with a high electric field will be called field selectivity and the selective condensation at the active centers of a structural origin will be called structural selectivity.

Since the growth surface relief changes continuously during condensate formation, the degree of supersaturation changes continuously on the microlevel. Therefore, we can only consider the local stationarity of the process, which is taken to be a constant numerical value of $E_{c}$ and its constant position in the unchanged spectrum of the effective energies of possible chemical bonds. Strictly speaking, such stationarity conditions are satisfied only in a certain condensation time interval.

The interaction of chemically active gases with the growth surface changes the energy spectrum of chemical bonds and, hence, the selective processes; therefore, a more reproducible result can be obtained in a pure inert atmosphere. We purified argon using the technique in [12] and minimized the partial pressures of chemically active gases to $8 \times 10^{-8} \mathrm{~Pa}$.

As a result, we state that high-porosity architectural forms at the plasma-condensate interface can only be formed via atom-by-atom assembling, at least under conditions of temporal local stationarity. To obtain a reproducible result, one must use a sufficiently pure working gas, and the formation of bulk architectural forms requires a proper volume diffusion field, i.e., use of a high working-gas pressure.

Our approach is based on process self-organization, which, in turn, is based on a low-temperature glow discharge stabilized by the magnetron effect [7] along with (in some cases) the hollow cathode effect $[8,9]$. The possibility of condensate formation at a high working-gas pressure $(\sim 20 \mathrm{~Pa})$ and the same potential of the sputtered target and the substrate holder are key factors.

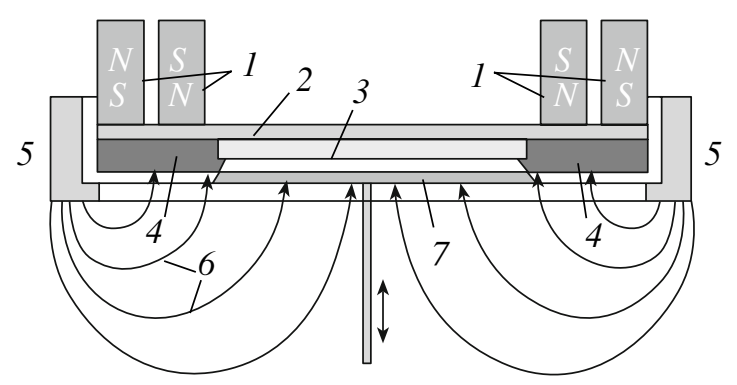

Fig. 1. Cross section of the sputtering system: (1) sputterer magnetic system, (2) water-cooled substrate and target holder, (3) substrate, (4) sputtered target, (5) anode, (6) force lines of the electric field, and (7) shutter.

In this work, we use a system designed on the basis of a dc magnetron sputterer with cylindrical symmetry (Fig. 1). Once argon is subjected to deep purification and shutter 7 is open, the reverse diffusion fluxes of the sputtered substance condense onto substrate 3. The main sputtered substance flux is focused on the substrate due to the directivity of force lines of electric field 6 and a relatively high working gas pressure. It should be noted that no more than one highly stabilized power supply in the system allows self-organization of all the technological parameters (the growth surface temperature and the intensities of the deposited flux and the plasma action) required for near-equilibrium condensation. A synchronous change in the technological parameters when the discharge power is varied in certain limits favors a self-organized stationary process. For example, as the discharge power increases, the deposited flux intensity, the growth surface temperature, and the plasma flux intensity increase simultaneously. In this case, the interrelated total changes in the technological parameters can lead to an unchanged condensation coefficient and constant $E_{c}$. Thus, not only near-equilibrium conditions are self-organized in systems like that considered, but also the process becomes stationary. The condensation mechanism can be changed using controlled parameters such as the discharge power and the working-gas pressure.

When our setup is used, reevaporated adatoms are, as a rule, repeatedly ionized and are again directed to the growth surface under the action of an electric field [13]. Therefore, a ring transfer mechanism near the substrate not only enhances the volume diffusion field, but also allows us to rationally use the sputtered material, increasing the condensate growth rate.

The aluminum layers were formed on glass substrates and were studied by scanning electron microscopy and X-ray diffraction.

\section{RESULTS AND DISCUSSION}

We performed two series of experiments and investigated the structure formation mechanisms in the aluminum condensates depending on the predominant 

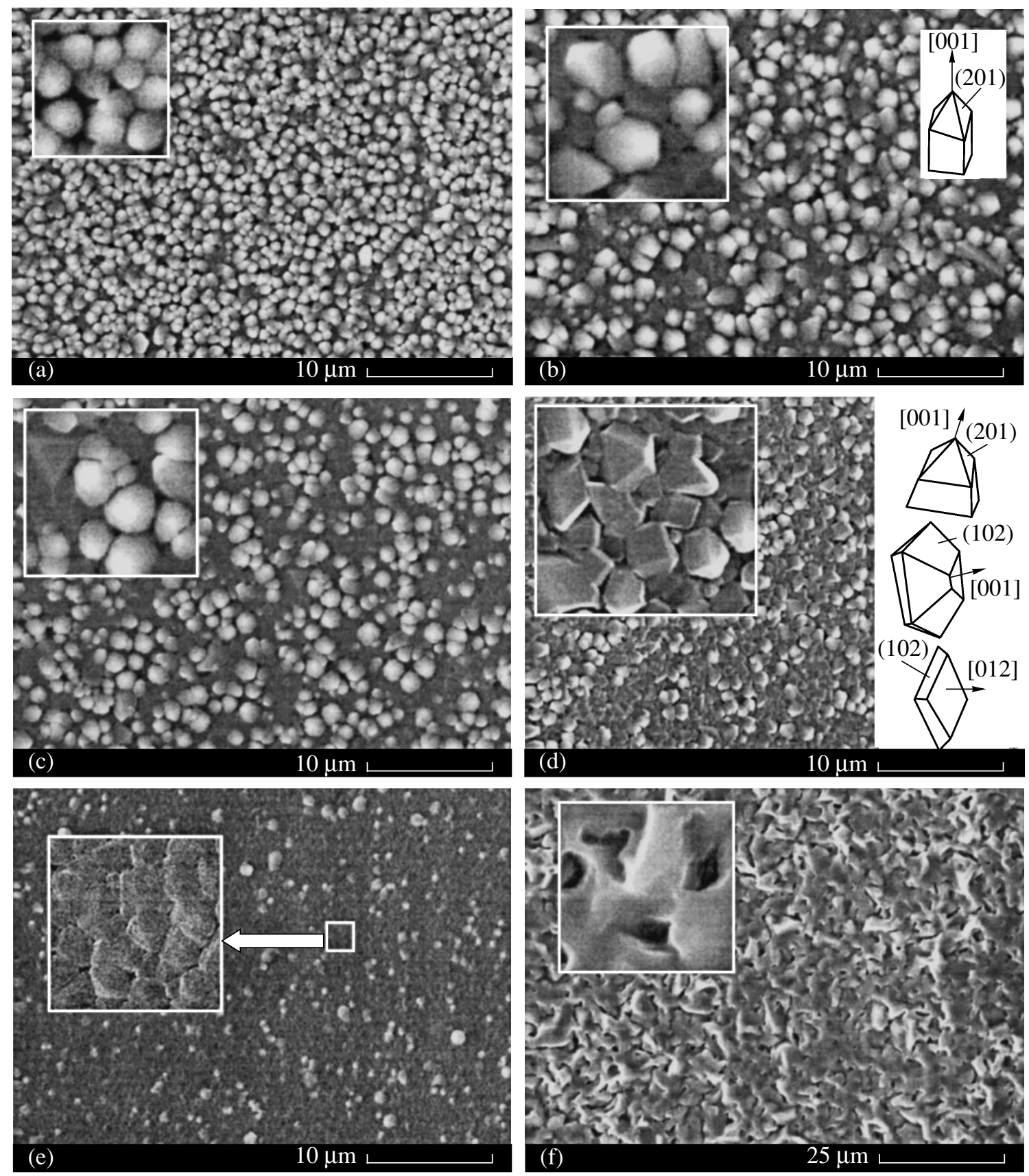

Fig. 2. Microstructures of the aluminum condensates formed as a result of the structural selectivity: (a) $P=2.4 \mathrm{~W}, P_{\mathrm{Ar}}=20 \mathrm{~Pa}$; (b) $P=2.6 \mathrm{~W}, P_{\mathrm{Ar}}=20 \mathrm{~Pa}$; (c) $P=6.4 \mathrm{~W}, P_{\mathrm{Ar}}=20 \mathrm{~Pa}$; (d) $P=6.4 \mathrm{~W}, P_{\mathrm{Ar}}=25 \mathrm{~Pa}$; (e) $P=6.4 \mathrm{~W}, P_{\mathrm{Ar}}=20 \mathrm{~Pa}$ (initial stage of condensate growth); and (f) $P=10.9 \mathrm{~W}, P_{\mathrm{Ar}}=20 \mathrm{~Pa}$.

contribution of the structural or field selectivity. It is obvious that the field selectivity should manifest itself at high discharge voltages (powers) and low workinggas pressures. For example, a change in the technological parameters in this direction, on the one hand, increases the electric field strength over the growth surface and, on the other hand, increases the ion free path. As a result, the trajectory of ion motion is more effectively changed in an electric field.

We first consider the laws of changes in the structure of the condensates formed as a result of the structural selectivity. Figure 2 shows a number of microstructures formed at the lowest discharge powers $(P=2.4-10.9 \mathrm{~W})$ and relatively high working-gas pressures $\left(P_{\mathrm{Ar}}=20-\right.$ $25 \mathrm{~Pa})$ on a water-cooled substrate holder. When analyzing these microstructures (Figs. 2a-2c), we conclude that the average crystallite size increases from 1 to $2 \mu \mathrm{m}$ provided that the discharge power increases from 2.4 to $2.6 \mathrm{~W}$. However, an increase in the discharge power from 2.6 to $6.4 \mathrm{~W}$ affects the structure formation only weakly (Figs. 2b, 2c), which supports the assumption about unchanged stationarity within 
certain limits of the discharge power. An analysis of the microstructures also indicates two stages in their formation, which differ in time. At the initial stage, the layer of weakly coupled nanocrystals forms (Fig. 2e) and the texture of their nucleation is controlled by the parallelism between the substrate surface and the (111) and (001) aluminum planes (Fig. 3a).

The presence of two nucleation textures seems to be natural, since, under the Volmer-Weber conditions for the aluminum-glass system and near-equilibrium conditions (high values of $E_{c}$ ), the binding energy of aluminum atoms on the substrate surface is maximal when it is covered by close-packed planes.

The transition to the second stage is accompanied by rapid condensate growth in the [001] direction (Fig. 3b) that yields the same habit plane of crystals based on the (210) plane (Fig. 2b).

To understand the change in the structure formation mechanisms, we estimate the effective energy spectrum of chemical bonds for aluminum atoms in different crystallographic positions for the situation of a semicrystal. Let this energy be $E_{(h k l)}$ for a certain $(h k l)$ plane. Obviously, $E_{(h k l)}$ is proportional to the number of the neighboring atoms of the $i$ th order $Z_{i}$. For aluminum (fcc lattice), the set of $Z_{1}, Z_{2}$, and $Z_{3}$ is given in the table.

As follows from these data, under near-equilibrium conditions, the structure continues forming exclusively on the basis of the (210) plane. In other words, the transition to the second stage of condensate growth is controlled by a decrease in the supersaturation; as a result, the value of $E_{c}$ is specified by the inequality $E_{(210)}>E_{c}>$ $E_{(531)}$ (see table). The supersaturation can be decreased via an increase in the growth surface temperature $T_{c}$ when the heat removal from this surface decreases upon an increase in the condensate thickness. It should be noted that the conditions that are rather close to equilibrium exclude repeated nucleation and favor the degradation of the crystal faceting (Figs. 2a, 2c). Eventually, such conditions premise the formation of columnar structures.

An increase in the working gas pressure to $25 \mathrm{~Pa}$, with other technological parameters remaining the same, enhances the reverse diffusion flux, which increases the supersaturation. This finding is confirmed by the weakening of the condensate texture (Fig. 3c) and by more pronounced crystal faceting (Fig. 2d). Condensation at higher pressures leads to the smearing of the boundary between two stages of condensate growth and favors secondary nucleation. Eventually, this behavior results in the formation of aggregates of weakly coupled crystals. In this case, the closeness to equilibrium manifests itself in the fact that almost the entire growth surface is based on the (210) plane (Fig. 2d).

As the discharge power increases to $10.9 \mathrm{~W}$, the pore formation mechanism changes radically (Fig. 2f). X-ray diffraction data demonstrate that, in contrast to the structure formation versions considered above, the two types of nucleation texture (Fig. 3a) remain unchanged

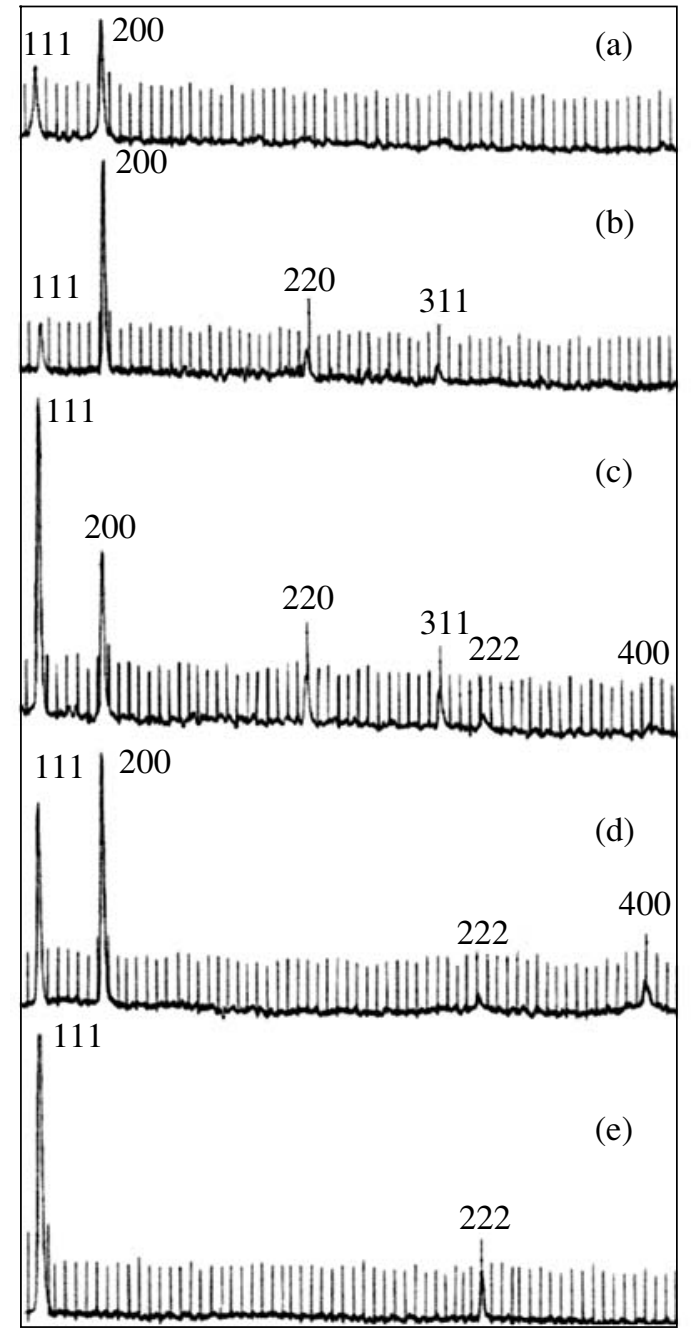

Fig. 3. X-ray diffraction patterns of the structures shown in Figs. 2 and 4: (a) Fig. 2e, (b) Figs. 2a-2c, (c) Fig. 2d, (d) Fig. 2f, and (e) Figs. 4a and 4b.

over all following stages of condensate formation (Fig. 3d). Therefore, the transition from a columnar to a labyrinth structure can be explained by different crystal-growth directions and subsequent association. To understand the related processes, we studied the stages of condensate formation for the presence of only one nucleation texture ((111) II substrate surface), which was formed when the substrate holder was not cooled.

Since the (111) plane is characterized by the highest degree of packing, a decrease in the supersaturation (an increase in the value of $E_{c}$ ) should inevitably lead to a preferred texture (Fig. 3e). As follows from the micro-

Values of $Z_{i}$ for various crystallographic planes in aluminum [3]

\begin{tabular}{c|c|c|c|c|c|c}
\hline Plane & $(210)$ & $(531)$ & $(311)$ & $(110)$ & $(100)$ & $(111)$ \\
\hline$Z_{1} Z_{2} Z_{3}$ & 6210 & 630 & 5310 & 5210 & 412 & 339 \\
\hline
\end{tabular}



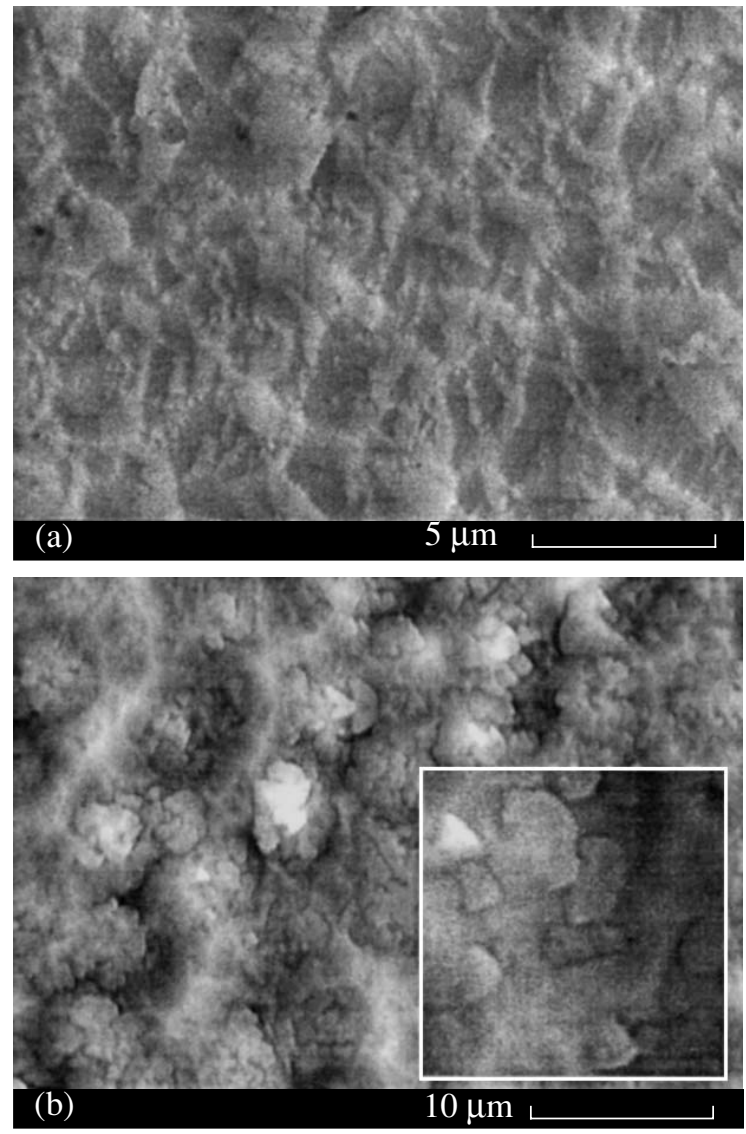

Fig. 4. Microstructures of the (a) initial and (b) final stages of the formation of aluminum condensates on an uncooled substrate holder $\left(P_{w}=6.4 \mathrm{~W}, P_{\mathrm{Ar}}=20 \mathrm{~Pa}\right)$.

structures shown in Figs. $4 \mathrm{a}$ and $4 \mathrm{~b}$, this version of nucleation texture substantially complicates the transition to pore formation in spite of a developed growth surface. This structure formation is likely to be controlled by the small values of $E_{(111)}$ (see table), i.e., the inequality $E_{c} \gg E_{(111)}$. In this case, the growth of the condensate nucleated on an unheated substrate via the incorporation of atoms in the (111) plane is excluded. Therefore, the necessary condition for condensate formation consists in the bending of the growth surface due to the appearance of crystallographic planes with higher values of $E_{(h k l)}$. As follows from the microstructure shown in Fig. 4 b, the crystals that are mainly oriented parallel to the substrate surface nucleate and grow at the final stage of structure formation. Among the possible causes of this directed growth, we can note the formation of the habitus of crystals on the basis of the (210) plane, which was shown to effectively nucleate on the (001) plane (see Figs. 2b, 2d). It is natural that, at higher degrees of supersaturation, this growth version is more pronounced, which is likely to result in the formation of the labyrinth structure shown in Fig. 2f.

We now analyze the experimental results obtained for the structure formation under the conditions of pos- sible field selectivity, i.e., at relatively low working-gas pressures $(\sim 1 \mathrm{~Pa})$ and the discharge power varying in the range $1.8-72.0 \mathrm{~W}$. When systematizing the spectrum of the fabricated structures, we were able to distinguish characteristic structures (Figs. 5a-5e) and detect the manifestation of the field selectivity. At relatively low discharge powers (1.8-2.4 W), i.e., in the absence of high electric fields, the condensate formation mechanism can be explained by the structural selectivity. This conclusion is supported by the crystallographic faceting of the structural elements and the absence of developed parts projecting from the growth surface (Figs. 5a, 5b). As in the cases considered above, condensates nucleate via the filling of the (111) and (001) aluminum planes parallel to the substrate surface. The specific features of the structure formed at the minimum discharge power (Fig. 5a) can be explained by chain nucleation of crystals on active centers in the growth surface. An analysis of the nucleation and growth texture and the character of the structural porosity (Fig. 5b) corroborates the assumption that the formation of the three-dimensional labyrinth structure is based on the association of crystals growing in different directions.

As the discharge power increases to $3.4 \mathrm{~W}$, important changes, which are related to the formation of a mesaporous structure without any signs of crystallographic faceting, occur in the structure formation mechanism (Fig. 5c). The absence of crystallographic faceting can be explained by the extreme closeness to equilibrium in the plasma-condensate system and by the corresponding fulfillment of the inequality $E_{c} \geq E_{(210)}$. In this case, the free energy of the growth monostep, which is specified by the relation $\alpha_{i}=n\left(E_{1}-k T_{c} \ln \left(1+2 \exp \left(-E_{1} / k T_{c}\right)\right)\right)$ (where $E_{1}$ is half the binding energy between the neighboring first-order atoms and $n$ is the total number of uncompensated bonds per unit length of the atomically smooth monostep), vanishes [3]. When the sign of $\alpha_{i}$ changes, growth monosteps become diffuse even in the (210) plane, which eventually causes the transition from the layer-by-layer growth of faceted crystals to their normal growth via condensation on an atomically rough surface [3].

Based on the $\alpha_{i}\left(T_{c}\right)$ curves plotted for various $E_{1}$ (Fig. 6), we found that the sign of $\alpha_{i}$ in the condensation temperature range slightly below the melting temperature of aluminum changes at $E_{1}=0.05 \mathrm{eV}$. This obviously low value of $E_{1}$ for this transition supports the necessity of using the effective chemical bond energies. Moreover, the replacement of tangential crystal growth by normal crystal growth excludes anisotropy in the growth rate in different crystallographic directions. These conditions are likely to minimize manifestation of the structural selectivity and favor the healing of macropores up to mesoaggregates (Fig. 5c). It should be noted that the decrease in the pore sizes is stimulated by a decrease in the condensate surface energy.

One of the important specific features of the structure shown in Fig. 5c is represented by the rapid growth 

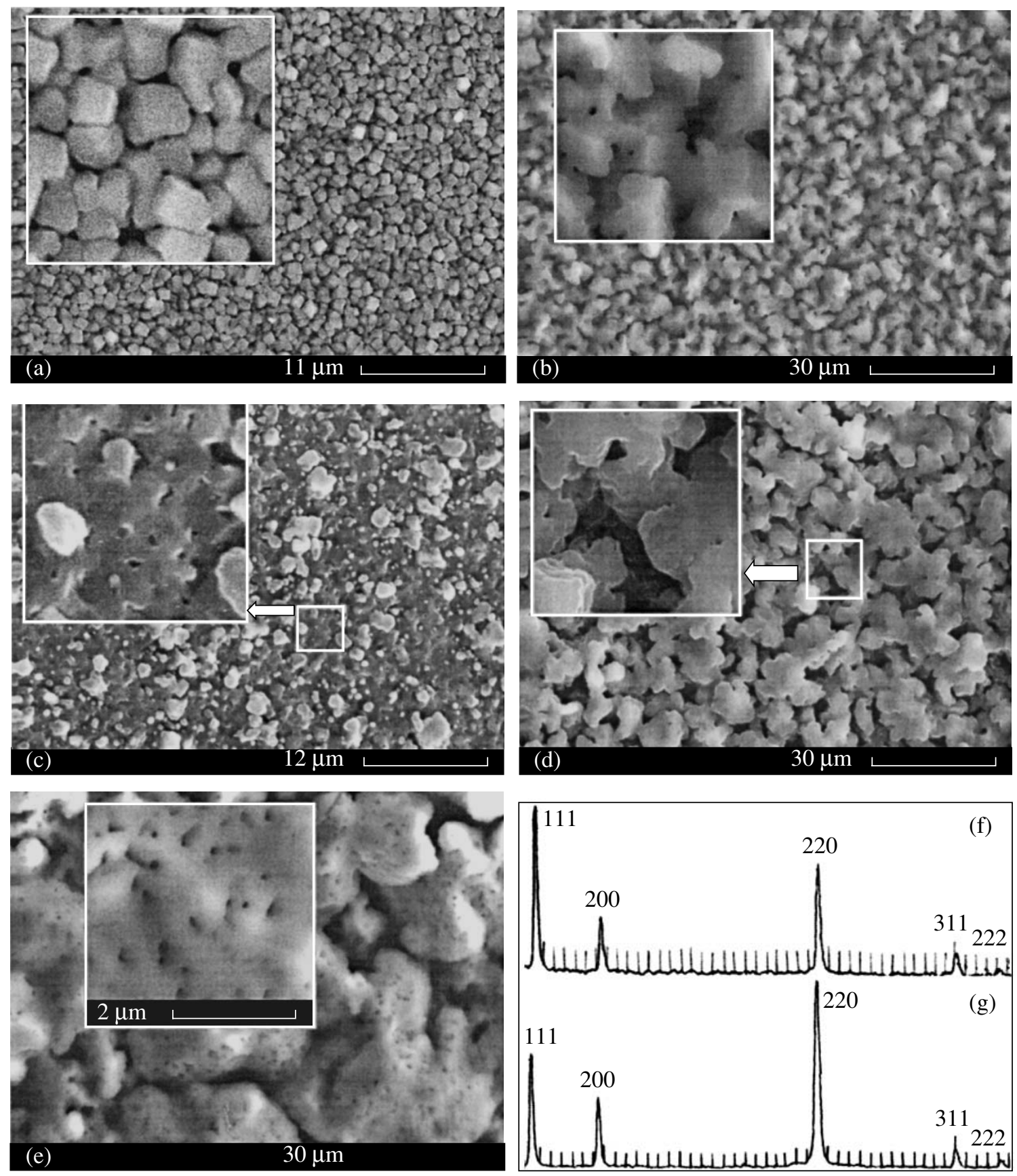

Fig. 5. Microstructures of the aluminum condensates formed as a result of the structural and field selectivity at $P_{\mathrm{Ar}}=1 \mathrm{~Pa}$ and $P=$ (a) 1.8, (b) 2.4, (c) 3.4, (d) 10.4, and (e) $72 \mathrm{~W}$, (f, g) X-ray diffraction patterns of the structures given in Figs. 5d and 5e, respectively.

of the projecting parts in the condensate induced by the field selectivity, and this tendency is enhanced as the discharge power increases to $10.4 \mathrm{~W}$. For example, the formation of the developed macropore structure shown in Fig. 5d in the mesaporous layer is mainly caused by the field selectivity, which is confirmed by the absence of crystallographic faceting and a pronounced columnar structure. A further increase in the discharge power to $72 \mathrm{~W}$ leads to the formation of a structure consisting of coarse (up to $40 \mu \mathrm{m}$ ) weakly coupled mesoporous polycrystals (Fig. 5e). Such structures are assumed to form via the association of macroporous fragments when passing to the normal crystal growth noted above. In this case, the role of the field selectivity is likely to be restricted to the coarsening of polycrystals.

The field selectivity also manifests itself in the enhancing of the crystal growth in the [011] direction (Figs. 5f, 5g).

In conclusion, we would like to note that the microstructures studied in this work do not reflect all the pos- 


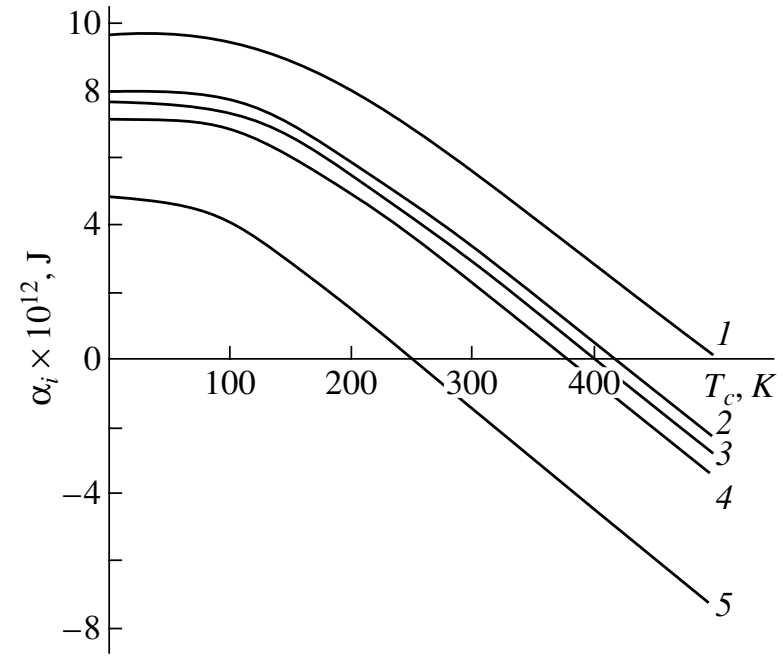

Fig. 6. $\alpha_{i}\left(T_{c}\right)$ curves plotted at $E_{1}=(1) 0.06$, (2) 0.05 , (3) 0.048, , (4) 0.045 , and (5) $0.03 \mathrm{eV}$.

sibilities of self-organized sputterers. It appears that a combination of the structural and field selectivities at a controlled change in the stationarity conditions can result in the atom-by-atom assembling of an unlimited spectrum of architectural forms, including important nanosystems. From this standpoint, self-organized ion sputterers can play a key role in the development of the novel nanotechnological field.

\section{CONCLUSIONS}

(1) Selective processes and the related pore formation are observed when $E_{c}$ is comparable with the maximum effective energy $E_{(210)}$, which supports the fact that the plasma-condensate system is close to equilibrium.

(2) The pore formation mechanisms in one technological cycle are based on sequential changes in the stationarity conditions, which are determined by a decrease in the supersaturation. The condensation mechanisms change when the critical energy is equal to the values of $E_{(h k l)}$.
(3) The transition from layer-by-layer to normal crystal growth and the related decrease in the surface energy promotes the healing of macropores up to mesoaggregates.

\section{REFERENCES}

1. P. Yu. Apel', V. V. Berezkin, A. B. Vasil'ev, et al., Membrany 3 (31), 45 (2006).

2. V. A. Malyshevskii and B. V. Farmakovskii, in Proceedings of the Kharkov Nanotechnology Assambly, Kharkov, 2007, p. 88.

3. A. A. Chernov, E. I. Givargizov, Kh. S. Bagdasarov, et al., in Modern Crystallography, Vol. 3: Crystal Growth, Ed. by B. K. Vainshtein (Nauka, Moscow, 1980).

4. L. I. Maissel and R. Glang, Handbook of Thin Film Technology (McGraw-Hill, New York, 1970; Sov. Radio, Moscow, 1977), Vol. 1.

5. L. S. Palatnik, M. Ya. Fuks, and V. M. Kosevich, Formation Mechanism and Substructure of Condensed Films (Nauka, Moscow, 1972) [in Russian].

6. S. A. Kukushkin and A. V. Osipov, Usp. Fiz. Nauk 168 , 1083 (1998) [Phys. Usp. 41, 983 (1998)].

7. V. I. Perekrestov, A. S. Kornyushchenko, and Yu. A. Kosminskaya, Pis'ma Zh. Tekh. Fiz. 32 (20), 1 (2006) [Tech. Phys. Lett. 32, 868 (2006)].

8. V. I. Perekrestov, Pis'ma Zh. Tekh. Fiz. 31 (19), 41 (2005) [Tech. Phys. Lett. 31, 830 (2005)].

9. V. I. Perekrestov and Yu. A. Kosminskaya, Pis'ma Zh. Eksp. Teor. Fiz. 78, 258 (2003) [JETP Lett. 78, 223 (2003)].

10. A. S. Bakai and V. E. Strel'nitskii, Structure and Physical Properties of Carbon Condensates Obtained by Deposition of Fast Particle Fluxes (TsNIIAtomInform, Moscow, 1984) [in Russian].

11. J. P. Hirth, S. J. Hruska, and G. M. Pound, in SingleCrystal Films, Ed. by M. H. Francombe and H. Sato (Macmillan, New York, 1964; Mir, Moscow, 1966).

12. V. I. Perekrestov and S. N. Kravchenko, Prib. Tekh. Eksp. 3, 123 (2002).

13. A. G. Zhiglinskii and V. V. Kuchinskii, Mass Transfer at Plasma-Surface Interaction (Energoatomizdat, Moscow, 1991) [in Russian].

Translated by K. Shakhlevich 